\title{
Surface Current Modelling of the Skin Effect for On-Chip Interconnections
}

\author{
Daniël De Zutter, Fellow, IEEE, Hendrik Rogier, Senior Member, IEEE, Luc Knockaert, Senior Member, IEEE, \\ and Jeannick Sercu
}

\begin{abstract}
In this paper, the skin effect for 2-D on-chip interconnections is predicted using a recently developed differential surface admittance concept. First, the features of the new approach are briefly recapitulated and details are given for a conductor with rectangular cross-section. Next, the 1-D situation is studied as a limiting case of the 2-D situation. The relationship with a local impedance formulation is investigated and illustrated with a numerical example. Finally, the new method is used to determine inductance and resistance matrices of 2-D on-chip interconnect examples with specifications taken from the International Technology Roadmap for Semiconductors. Extra capacitance data are also provided.
\end{abstract}

Index Terms-On-chip interconnect, resistance and inductance matrices, skin effect, surface impedance/admittane.

\section{INTRODUCTION}

$\mathbf{T}$ HE EVOLUTION towards smaller chip features and increasing clock rates continues as the International Technology Roadmap for Semiconductors (ITRS) ${ }^{1}$ predicts that the smallest on-chip features will shrink from $150 \mathrm{~nm}$ in 2003 to $50 \mathrm{~nm}$ by 2012 while the clock rate will increase from 1.5 to 10 GHz. An important issue in the representation of signal conductors and their coupling is the correct modelling of the so-called skin effect, also known as current crowding; see, e.g., [1]-[3]. In [4], a new differential surface admittance description for good conductors was proposed. At each frequency, this surface admittance description associates a fictitious electric surface current density $\mathbf{J}_{s}(\mathbf{r}, \omega)$ at each point $\mathbf{r}$ on the surface of the conductor to the tangential electric fields $\mathbf{E}_{t}\left(\mathbf{r}^{\prime}, \omega\right)$ at every other point on the surface. The surface admittance description allows one to replace a conductor by equivalent electric surface currents and to replace the conductor medium by the medium of the material layer in which it is embedded. The remaining field problem can then be solved by solely considering the interactions between the equivalent surface currents.

Manuscript received September 20, 2006; revised November 4, 2006. This work was supported in part by the Institute for the Promotion of Innovation by Science and Technology, Flanders, for a joint project with Agilent Technologies. The work of H. Rogier was supported by DWTC/SSTC under the MOTION Project.

D. De Zutter, H. Rogier, and L. Knockaert are with the Department of Information Technology, Ghent University, B-9000 Ghent, Belgium (e-mail: daniel. dezutter@ugent.be).

J. Sercu is with Agilent Technologies Belgium, B-9051 Ghent, Belgium (e-mail: jeannick_sercu@agilent.com).

Digital Object Identifier 10.1109/TADVP.2007.895984

${ }^{1}$ http://www.public.itrs.net
Section II very briefly recapitulates the general idea behind the differential admittance concept as derived in [4], restricting ourselves to 2-D configurations and to the transverse magnetic (TM) case, for which the electric field is directed along the longitudinal $z$-axis and the magnetic field only has components in the transversal $(x, y)$-plane. In [3], the TM case is treated using a finite difference solution of the Helmholtz equation in the conductor's cross-section. Here, a general solution is obtained in terms of the Dirichlet eigenfunctions of the cross-section. Special attention is devoted to a conductor with rectangular cross-section.

In Section III, the exact 2-D theory for a conductor with rectangular cross-section (width $w$, thickness $t$ ) is used to derive a 1 -D approximation for thin conductors, i.e., for $t \ll w$. Note that "thin" only implies that the width is much larger than the thickness but does not imply that the conductor is thin with respect to the skin depth $\delta=\sqrt{2 / \omega \mu_{0} \sigma}$. In the 1-D approximation, fictitious electric surface currents at the top and bottom of the conductor, both depending on the electric field at the top and at the bottom of the conductor, describe the conductor's behavior over the complete frequency range. This approximation is compared to the usual surface impedance of a conductor of thickness $t$ [5]-[8] and illustrated for a conductor above a ground plane.

Section IV briefly states how the equivalent surface current can be used to determine the resistance and inductance matrices of a set of parallel conductors by means of an electric field integral equation (EFIE). This is followed by a set of numerical examples, intended to illustrate the capability of the method to handle realistic on-chip configurations. These examples are derived from the CODESTAR (compact modelling of on-chip passive structures at high frequencies) IST [9] project and take into account the most recent specifications of the ITRS. Four examples discuss various configurations in which a single signal conductor is surrounded by "ground" conductors. A fifth example considers coupled signal lines. To complete the transmission-line model, additional capacitance data are also provided.

\section{Equivalent Surface CuRRent AND SuRface ADMITTANCE}

We restrict ourselves to the time-harmonic ( $e^{j \omega t}$ dependence) TM polarization. $E_{z}$ is the electric field inside the homogeneous nonmagnetic conductor with constitutive parameters $\epsilon$, $\mu_{0}$, and $\sigma$, and cross section $S$. The conductor is embedded in a planar stratified medium, and the nonconducting and nonmagnetic layer in which the conductor is embedded is characterized 
by the constitutive parameters $\epsilon_{\text {out }}$ and $\mu_{0}$. On the boundary $c$ of $S$, we have that

$$
H_{t}=\frac{1}{j \omega \mu_{0}} \frac{\partial E_{z}}{\partial n}
$$

with the index $t$ referring to the tangential component of the magnetic field and with $\partial E_{z} / \partial n$ representing the limit of the normal derivative of the electric field tending from the inside of the conductor to $c$. We replace the conducting medium by the medium outside the conductor, in particular the medium of the material layer in which the conductor is embedded. On the boundary $c$ of $S$, we now find

$$
H_{t 0}=\frac{1}{j \omega \mu_{0}} \frac{\partial E_{z 0}}{\partial n} .
$$

Consequently, the conductor can be replaced by the material of its surrounding layer, undoing the discontinuity in conductivity and permittivity due to the conductor's presence, by introducing an equivalent electric surface current density $J_{s}$ related to the value of the field $E_{z 0}$ on the boundary through the differential surface admittance operator $\mathcal{Y}$

$$
J_{s}=\mathcal{Y} E_{z 0}=\mathcal{Y} E_{z}=\frac{1}{j \omega \mu_{0}}\left(\frac{\partial E_{z}}{\partial n}-\frac{\partial E_{z 0}}{\partial n}\right) .
$$

Note that on $c$ and only on $c, E_{z 0}=E_{z}$ as the presence of the surface current $J_{s}$ only introduces a jump in the tangential magnetic field. When solving the field problem external to the conductor, the effect of the conductor is exactly accounted for by the presence of the surface current $J_{s}$, provided $E_{z}=E_{z 0}$ and that the surface current $J_{s}$ is forced to satisfy (3) on $c$. Inside the conductor, a fictitious field is obtained.

A general way to obtain the operator $\mathcal{Y}$ is to use the Dirichlet eigenfunctions of the cross section $S$. Calculations, the details of which are given in [4], show that

$$
J_{s}=\mathcal{Y} E_{z}=\tau \sum_{m=1}^{\infty} \frac{\frac{\partial \xi_{m}}{\partial n} \oint_{c} E_{z} \frac{\partial \xi_{m}}{\partial n} d c}{\left(k_{\text {out }}^{2}-\lambda_{m}\right)\left(k^{2}-\lambda_{m}\right)}
$$

with $\tau=\left[\sigma+j \omega\left(\epsilon-\epsilon_{\text {out }}\right)\right]$ and where $k$ is the wavenumber of the conductor and $k_{\text {out }}$ the wavenumber of the medium replacing the conductor. The $\xi_{m}$ are the Dirichlet eigenfunctions of the cross-section $S$ with corresponding eigenvalues $\lambda_{m}$.

The Joule losses associated with the surface current $J_{s}$ are

$$
\mathcal{P}_{s}=\Re\left\{\oint_{c} J_{s} E_{z}^{*} d c\right\}=\sigma \int_{S}\left|E_{z}\right|^{2} d S
$$

which are equal to the Joule volume losses. Moreover, the total surface current is

$$
\oint_{c} J_{s} d c=(\sigma+j \omega \epsilon) \int_{S} E_{z} d S-j \omega \epsilon_{\mathrm{out}} \int_{S} E_{z 0} d S .
$$

For a good conductor, the contribution of the displacement current in the right-hand side of (6) can be neglected, and the total surface current is quasi-identical to the total conduction current in the conductor.
For a rectangular conductor $(0 \leq x \leq w$ and $0 \leq y \leq t)$, the Dirichlet eigenfunctions and eigenvalues are

$$
\xi_{m n}=\frac{2}{\sqrt{w t}} \sin \left(\frac{m \pi x}{w}\right) \sin \left(\frac{n \pi y}{t}\right)
$$

with $\lambda_{m n}=((m \pi) / w)^{2}+((n \pi) / t)^{2}$. In [4], it is shown that an analytical expression for $J_{s}$ can be obtained by expanding $E_{z}$ on each side of the rectangle in an appropriate Fourier sine series. In Section IV, we will apply a method of moments (MoM) approach to obtain R- and L-matrices. To be able to do so, we need a discretized form of the operator $\mathcal{Y}$. To this end, both $E_{z}$ and $J_{s}$ are expanded in pulse basis functions along the four sides of the rectangle. All the pulse basis amplitudes $E_{j}$ for the four sides can be collected into a vector $\mathbf{E}$ and all the pulse basis amplitudes $J_{j}$ can be collected into a vector $\mathbf{J}$. Long, but completely analytical, calculations lead to the discretized analytical form of $\mathcal{Y}$

$$
\mathbf{J}=\mathbf{Y}_{s} . \mathbf{E} .
$$

$\mathbf{Y}_{s}$ is the surface admittance matrix. We refer the reader to [4] for detailed expressions of the elements of this surface admittance matrix.

\section{LOCAL SURfaCe IMPEDANCE APPROXIMATION}

When modeling finite thickness conductors for packaging applications, the full modeling of the interior of the conductor is often avoided by introducing a suitable local surface impedance model [5]-[8]. In that case, only the local thickness $t$ of the conductor is taken into account. Let us restrict this discussion to the case for which the conductor is modeled as a 3-D conductor, i.e., separate currents are introduced at the top and at the bottom of the conductor. For this situation, the following surface impedance is typically introduced and is expected to yield acceptable results:

$$
Z_{s}=\sqrt{\frac{j \omega \mu_{0}}{\sigma}} \operatorname{coth}\left(\sqrt{j \omega \mu_{0} \sigma} t / 2\right)
$$

with $\sigma \gg j \omega \epsilon$. At low frequencies, (9) reduces to $2 / \sigma t$. Hence, the total low-frequency resistance is half that value (two sheets in parallel), i.e., the correct dc value. At high frequencies, the coth-part in (9) tends to unity and (9) becomes $(1+j) / \sigma \delta$, with $\delta=\sqrt{2 / \omega \mu_{0} \sigma}$ the skin depth. This is what we expect: the current runs in two skin depth layers, one at each side of the conductor.

Let us now turn to the exact surface admittance result for the rectangle to find out what this result can tell us about the above surface impedance model (9). To this end, we need the following analytical result from [4]. For a rectangle of dimensions $w$ by $t$, the electric field $E_{z}^{-}$on the bottom side, the electric field $E_{z}^{+}$ on the top side, the differential surface admittance current $J_{s}^{-}$ on the bottom side, and its counterpart on the top side $J_{s}^{+}$are all expanded in a Fourier sine-series of the form

$$
\sum_{m=1}^{\infty} A_{m} \sin \frac{m \pi x}{w} .
$$


Let us now first focus on the contribution of $E_{z}^{-}$to the surface currents $J_{s}^{-}$and $J_{s}^{+}$. If $E_{z m}^{-}$is the amplitude of the $m$ th term in the expansion of $E_{z}^{-}$, the corresponding amplitudes for the currents are

$$
\begin{aligned}
& J_{s m}^{-}=\frac{2 \sigma t}{\pi^{2}} \sin (m \pi x / w) \Omega^{\prime}\left(0, p_{m}, q_{m}\right) E_{z m}^{-} \\
& J_{s m}^{+}=-\frac{2 \sigma t}{\pi^{2}} \sin (m \pi x / w) \Omega^{\prime}\left(\pi, p_{m}, q_{m}\right) E_{z m}^{-}
\end{aligned}
$$

with

$$
\Omega(u, p, q)=\sum_{j=1}^{\infty} \frac{j \sin (j u)}{\left(p^{2}-j^{2}\right)\left(q^{2}-j^{2}\right)}
$$

and

$$
p_{m}=\frac{t}{\pi} \sqrt{k^{2}-\left(\frac{m \pi}{w}\right)^{2}}, \quad q_{m}=\frac{t}{\pi} \sqrt{k_{\text {out }}^{2}-\left(\frac{m \pi}{w}\right)^{2}} .
$$

$\Omega^{\prime}(u)$ stands for the derivative of $\Omega(u)$ with respect to its argument $u$. The function $\Omega$ is given by

$\Omega(u, p, q)=-\frac{\pi}{2}\left[\frac{\sin (\pi p-p u)}{\left(q^{2}-p^{2}\right) \sin (\pi p)}+\frac{\sin (\pi q-q u)}{\left(p^{2}-q^{2}\right) \sin (\pi q)}\right]$

$$
0 \leq u<2 \pi \text {. }
$$

For further details, the reader is again referred to [4]. Remark that each term of the Fourier series for $E_{z}^{-}$only gives rise to the corresponding term in the Fourier series of the equivalent currents, i.e., no cross coupling between Fourier components occurs [see (11) and (12)]. The coupling between $E_{z m}^{+}$and the currents $J_{s m}^{-}$and $J_{s m}^{+}$can be described in a completely analogous and symmetric way.

If we now let $w \rightarrow \infty$, i.e., we consider a "thin" conductor $(t \ll w),(11)$ and (12) become

$$
\begin{aligned}
& J_{s m}^{-}=Y_{1} E_{z m}^{-} \\
& J_{s m}^{+}=Y_{2} E_{z m}^{-}
\end{aligned}
$$

with

$$
\begin{aligned}
& Y_{1}= \sqrt{\frac{\sigma}{j \omega \mu_{0}}} \operatorname{coth}\left(\sqrt{j \omega \mu_{0} \sigma} t\right) \\
&- \sqrt{\frac{\epsilon_{\text {out }}}{\mu_{0}}} \operatorname{coth}\left(k_{\text {out }} t\right) \\
& Y_{2}=-\left(\sqrt{\frac{\sigma}{j \omega \mu_{0}}} \frac{1}{\sinh \left(\sqrt{j \omega \mu_{0} \sigma} t\right)}\right. \\
&\left.-\sqrt{\frac{\epsilon_{\text {out }}}{\mu_{0}}} \frac{1}{\sinh \left(k_{\text {out }} t\right)}\right) .
\end{aligned}
$$

$\epsilon_{\text {out }}$ is the dielectric constant of the material of the layer in which the conductor is embedded and $k_{\text {out }}=\omega \sqrt{\epsilon_{\text {out }} \mu_{0}}$ is the wavenumber of that layer. The width $w$ no longer occurs in the above formulas. Taking into account that the above reasoning applies for each term in the Fourier series and that a similar reasoning holds for the contribution from $E_{z}^{+}$, the following surface admittance relationship is obtained:

$$
\left(\begin{array}{c}
J_{s}^{+} \\
J_{s}^{-}
\end{array}\right)=\left(\begin{array}{ll}
Y_{1} & Y_{2} \\
Y_{2} & Y_{1}
\end{array}\right)\left(\begin{array}{l}
E_{z}^{+} \\
E_{z}^{-}
\end{array}\right)=\mathbf{Y}_{1 D}\left(\begin{array}{l}
E_{z}^{+} \\
E_{z}^{-}
\end{array}\right) .
$$

$\mathbf{Y}_{1 D}$ is the 1-D counterpart of (8) and is again symmetric.

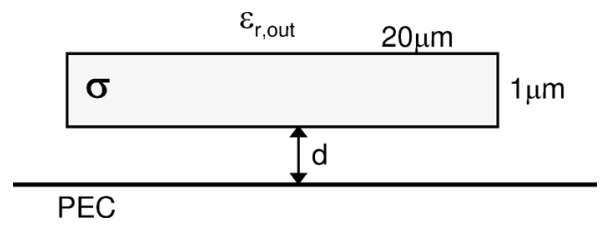

Fig. 1. Rectangular conductor above a PEC ground plane $\left(\sigma=5.7 \times 10^{7} \mathrm{~S} / \mathrm{m}\right.$, $\left.\epsilon_{r, \text { out }}=4\right)$.

Let us now take a closer look at the properties of $\mathbf{Y}_{1 D}$ and its relationship, if any, to the more simple expression (9). At low frequencies, we invoke the following series expansions of the hyperbolic functions in (18) and (19): $\operatorname{coth} x \approx 1 / x+x / 3$ and $1 / \sinh x \approx 1 / x-x / 6$ for small values of $x$. In that case, $\mathbf{Y}_{1 D}$ becomes

$$
\mathbf{Y}_{1 D}=\frac{\sigma d}{6}\left(\begin{array}{ll}
2 & 1 \\
1 & 2
\end{array}\right)
$$

Note that, in taking the limit for $\Omega \rightarrow 0$, the terms inversely proportional to the square root of $\sigma$ nicely cancel out. Moreover, at very low frequencies, a uniform current density will be found inside the conductor and consequently the currents at the top and bottom of the conductor will be identical $\left(J_{s}^{+}=J_{s}^{-}\right)$. This will also be the case for the electric fields $\left(E_{z}^{+}=E_{z}^{-}\right)$. Hence, at both the top and the bottom of the conductor, the boundary condition $J_{s}=\sigma d(2 / 6+1 / 6) E_{z}=(\sigma d / 2) E_{z}$ is enforced. This was also obtained from (9). Note, however, that (21) is more complex than (9) and tells us that when the electric field at top and bottom starts to become different (when the frequency increases), the correct surface admittance behavior is no longer captured by (9). This will also be clear from the example given below. In the same context, it is interesting to note that $Y_{1}, Y_{2}$, and $Z_{s}$ (9) exactly satisfy the relationship $Y_{1}+Y_{2}=1 / Z_{s}$. Hence, as long as the electric fields at the top and bottom of the conductor remain (approximately) identical, (21) and (9) will yield identical results.

At high frequencies, i.e., in the skin effect regime, the situation is quite simple. For $\sigma \gg \omega \epsilon_{\text {out }}$ (and provided the thickness $t$ remains sufficiently small with respect to the wavelength $\left.\lambda_{\text {out }}=2 \pi / k_{\text {out }}\right), \mathbf{Y}_{1 D}$ reduces to

$$
\mathbf{Y}_{1 D}=\sqrt{\frac{\sigma}{j \omega \mu_{0}}}\left(\begin{array}{ll}
1 & 0 \\
0 & 1
\end{array}\right)
$$

Currents at the top and bottom of the conductor are now decoupled, and we recuperate the well-known skin effect description, which also follows from (9).

Let us now turn to an example to illustrate the above theory. Fig. 1 shows a copper conductor $\left(\sigma=5.7 \times 10^{7} \mathrm{~S} / \mathrm{m}\right)$ with width $w=20 \mu \mathrm{m}$ and thickness $t=1 \mu \mathrm{m}$ placed in a homogeneous dielectric with $\epsilon_{r, \text { out }}=4$ at a distance $d$ above a perfectly electric conducting (PEC) ground plane. By choosing a width-to-thickness ratio of 20 , we are entitled to approximate the configuration by a conducting slab for which the currents on the vertical side become much less important and for which the approximate 1-D formulas discussed above can be applied. Figs. 2 and 3 display the normalized resistance per unit of length (PUL) for the configuration of Fig. 1 as a function of frequency and for $d=0.5$ and $2 \mu \mathrm{m}$, respectively. The frequency scales in 


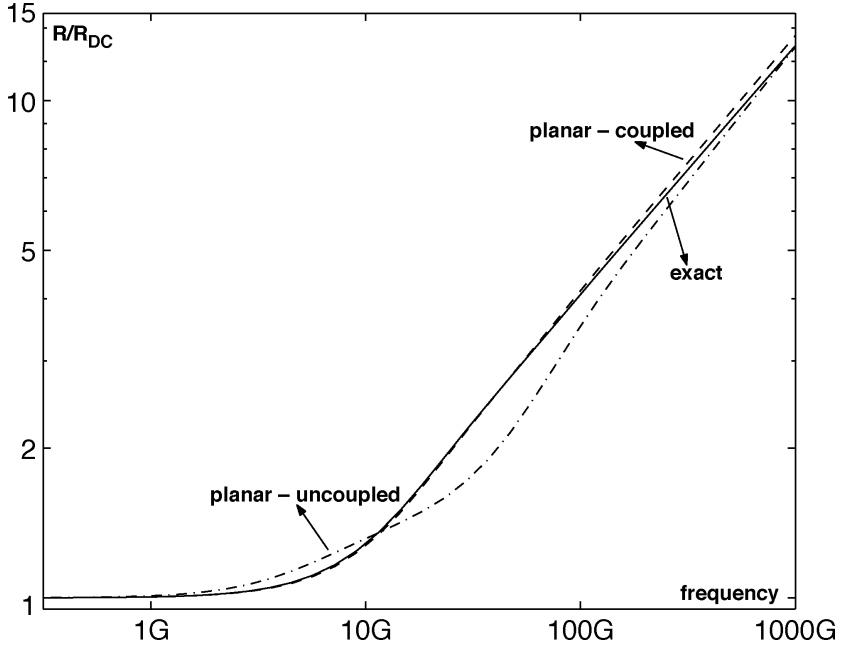

Fig. 2. Normalized resistance PUL as a function of frequency for the configuration of Fig. 1 and for $d=0.5 \mu \mathrm{m}$ [full line: exact 2-D result (8); dashed line: 1-D result with coupling between top and bottom (20); dashed-dotted line: 1-D result with no coupling between top and bottom (9)].

Figs. 2 and 3, as in all the figures that follow, have been chosen such that, at the lowest frequency used in the figure, the displayed data are still (almost completely) identical to their dc value (as verified by numerical calculations). These results were obtained by solving an integral equation for the surface current, as explained in the sequel (Section IV). At $0.5 \mathrm{GHz}$, the skin depth $\delta=2.981 \mu \mathrm{m}$ and becomes as small as $0.0687 \mu \mathrm{m}$ at $1000 \mathrm{GHz}$. The value of the resistance is normalized with respect to the dc value of $877.19 \Omega / \mathrm{m}$. On each figure three curves are displayed. The full line is the result obtained with the exact 2-D formulation of (8) (with 140 pulse functions along the width and seven pulse functions along the thickness, i.e., a total of 294 discretizations). The dashed line (planar-coupled) is the result obtained with the local coupling between top and bottom as described by (20). This implies that we have simplified the calculations by neglecting the currents on the vertical sides, as explained above. Finally, the dash-dot line (planar-uncoupled) is the corresponding result, whereby (9) is separately applied to both the current on the top and the current on the bottom. Figs. 2 and 3 confirm the theoretical expectations. At very low frequencies, all approaches yield the same result. They also tend towards the same result at high frequencies. The 1-D coupled description (20) remains much more accurate over the whole frequency range as compared to the 1-D uncoupled description (9). The difference between the results for $d=0.5$ and $2 \mu \mathrm{m}$ is that in the first case, due to the close proximity of the PEC plane, the electric field at top and bottom differ more than in the second case. As argued above, this will lead to a more prominent difference between the coupled and uncoupled approach, as clearly confirmed by the numerical results. Additional numerical data can be found in Figs. 4 and 5, where the corresponding inductances PUL are displayed, again normalized with respect to the $\mathrm{dc}$ value $(44.98 \mathrm{nH} / \mathrm{m}$ for $d=0.5 \mu \mathrm{m}$ and $110.76 \mathrm{nH} / \mathrm{m}$ for $d=2 \mu \mathrm{m}$ ). The same phenomena as for the resistances can be observed. However, neglecting the current on the vertical sides here already leads to an error of $1 \%-2 \%$ for the lowest frequencies.

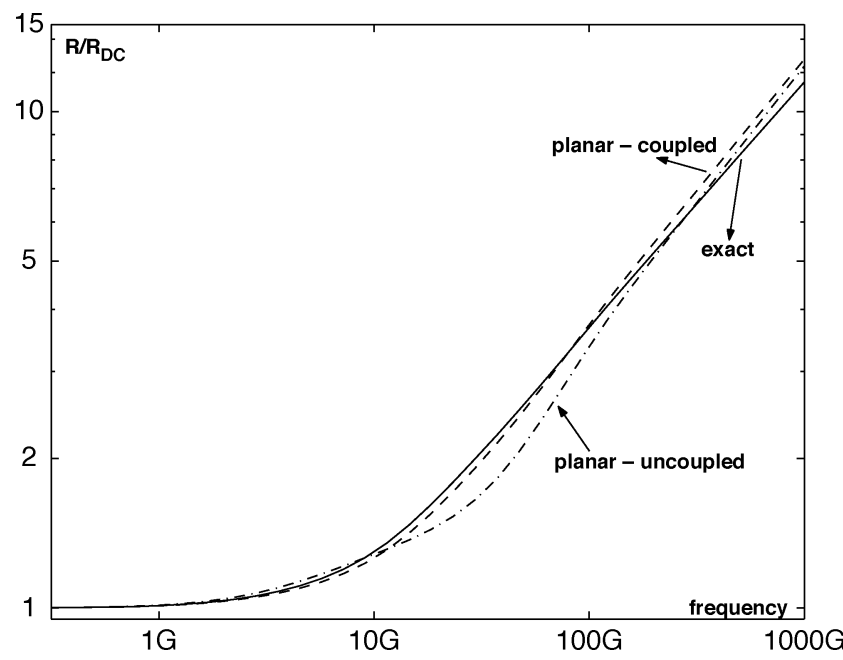

Fig. 3. Normalized resistance PUL as a function of frequency for the configuration of Fig. 1 and for $d=2 \mu \mathrm{m}$ [full line: exact 2-D result (8); dashed line: $1-D$ result with coupling between top and bottom (20); dashed-dotted line: 1-D result with no coupling between top and bottom (9)].

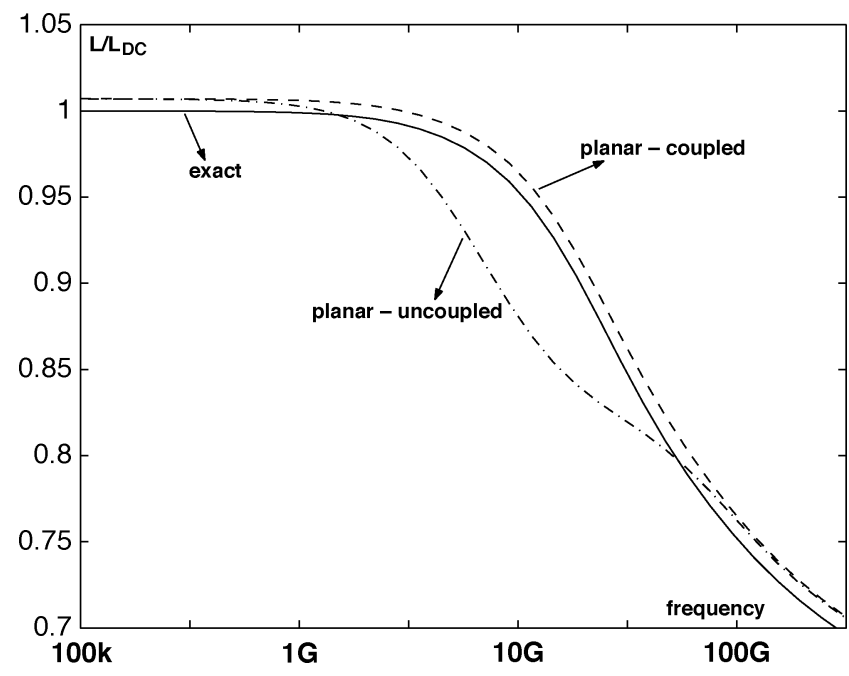

Fig. 4. Normalized inductance PUL as a function of frequency for the configuration of Fig. 1 and for $d=0.5 \mu \mathrm{m}$ [full line: exact 2-D result (8); dashed line: 1-D result with coupling between top and bottom (20); dashed-dotted line: 1-D result with no coupling between top and bottom (9)].

\section{RESistance AND INDUCTANCE MATRICES FOR ON-CHIP SIGNAL LINES}

In this section, we turn to the prediction of the behavior of a set of $N$ on-chip parallel signal lines, modeled as infinite 2-D multiconductor transmission lines. The relevant EFIE, valid as long as the cross-sectional dimensions remain small with respect to the wavelength in the nonmagnetic background medium, is [1]

$$
E_{z}(\mathbf{r})=-j \omega A_{z}(\mathbf{r})-\frac{\partial V(\mathbf{r})}{\partial z} .
$$

$A_{z}$ is the vector potential and $V$ is the scalar potential. Using the differential surface admittance concept, the conductors can be replaced by equivalent surface currents $J_{s}$. The vector potential of these currents is given by

$$
A_{z}(\mathbf{r})=-\mu_{0} \oint_{c} J_{s}\left(\mathbf{r}^{\prime}\right) \frac{1}{2 \pi} \ln \left|\mathbf{r}-\mathbf{r}^{\prime}\right| d c\left(\mathbf{r}^{\prime}\right) .
$$




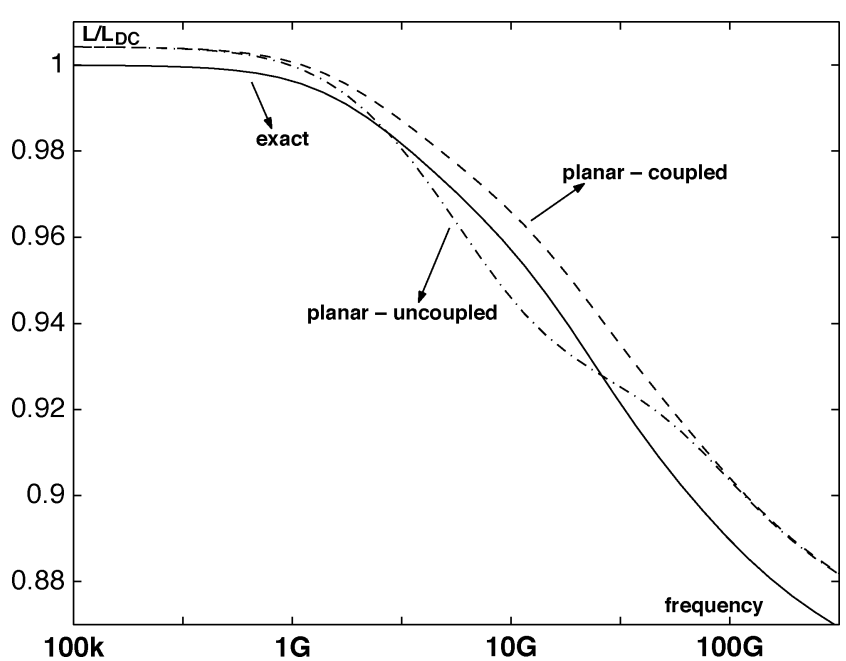

Fig. 5. Normalized inductance PUL as a function of frequency for the configuration of Fig. 1 and for $d=2 \mu \mathrm{m}$ [full line: exact 2-D result (8); dashed line: $1-\mathrm{D}$ result with coupling between top and bottom (20); dashed-dotted line: 1-D result with no coupling between top and bottom (9)].

As explained in [1], in the considered approximation, the dielectric contrast does not come into play and the voltage $V$ remains constant over the cross-section of each conductor (but of course still depends upon the longitudinal coordinate $z$ ). The relationship between these constant voltage values and the total currents through each conductor is

$$
\frac{\partial \mathbf{V}}{\partial z}=-(\mathrm{R}+j \omega \mathrm{L}) \mathbf{I}=-\mathbf{Z} \mathbf{I} .
$$

In (25), $\mathbf{V}$ is an $N \times 1$ column vector formed by the constant potentials $V_{p}$ of each conductor cross section, with $p=$ $1,2, \ldots, N . I$ is also an $N \times 1$ column vector formed by the total currents $I_{p}$ through each conductor $p$; R and L, respectively, represent the $N \times N$ resistance; and inductance matrix and $\mathrm{Z}$ is the impedance matrix. Substituting (24) and (25) into (23), discretizing the resulting integral equation with a Galerkin MoM using pulse basis functions and invoking (8) to express the relationship between the surface currents and the electric field, we can finally determine the matrices R and L. For more details the reader is referred to [4].

Let us now apply the above approach to a set of 2-D configurations as depicted in Fig. 6(a)-(d). These four examples are taken from the CODESTAR-IST project [9], with some modifications in the dimensions, and are representative of advanced on-chip interconnects as proposed by the ITRS roadmap . All cross-sectional dimensions are in nanometers, and the effective resistivity of the conductors is $2.2 \mu \mathrm{m} \cdot \Omega \cdot \mathrm{cm}$ (we have chosen a value between that of copper and Al-oxide, as proposed in [9]). In all cases, the dark shaded center conductor is taken to be the signal conductor and the other conductors are on zero, i.e., reference potential. Fig. 7 displays the resulting resistance PUL in $\Omega / \mathrm{m}$ between 1 and $1000 \mathrm{GHz}$. The corresponding inductances PUL in nanohenry/meter are shown in Fig. 8. In the MoM solution of (23)-(25), one pulse basis function per $13.5 \mathrm{~nm}$ was used, e.g., amounting to a total of 1020 unknown surface currents for the configuration of Fig. 6(a). To solve the problem of
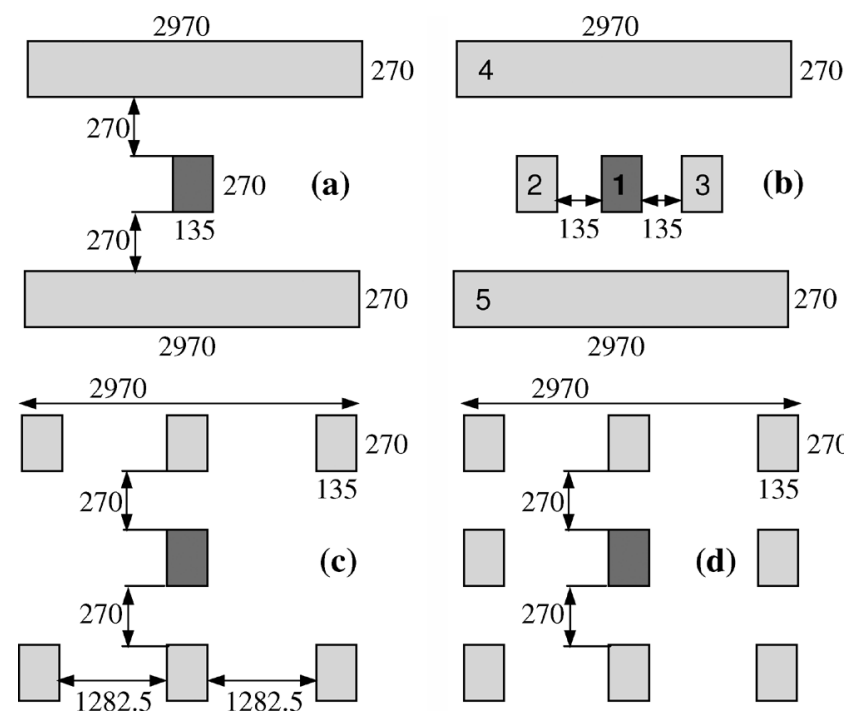

Fig. 6. On-chip signal line configurations: dark shaded central signal line surrounded by ground lines (all dimensions are in nanometers).

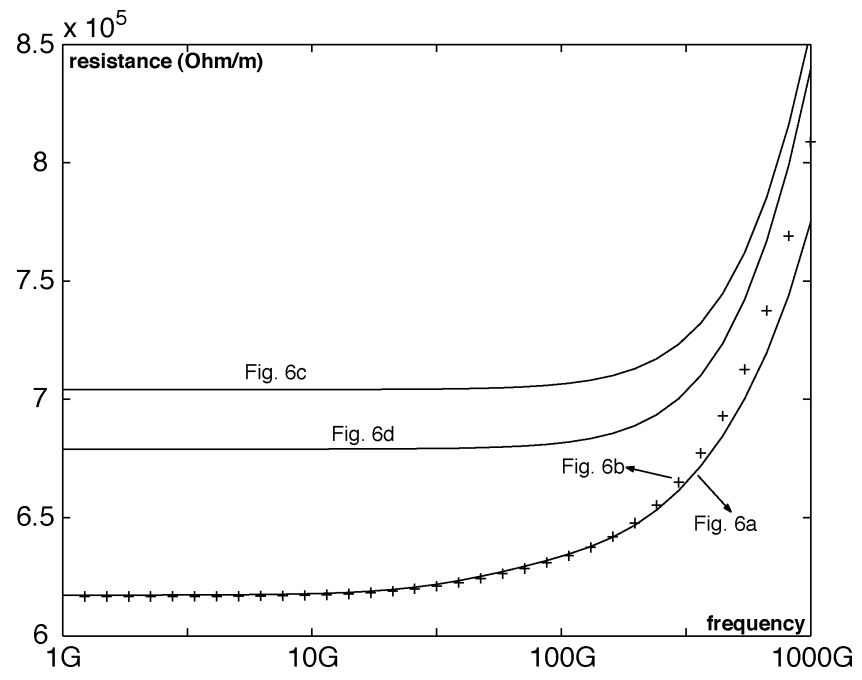

Fig. 7. Resistance PUL in $\Omega / \mathrm{m}$ as a function of frequency for the configurations of Fig. 6.

Fig. 6(b), we use the theory put forward in Section II to first calculate the $960 \times 960$ surface impedance matrices $(8)$ of the two large conductors and the corresponding $60 \times 60$ matrices of the three small conductors. A similar reasoning of course applies to the other configurations. The results of Figs. 7 and 8 cannot be obtained directly from the solution of the integral equation. To find the results for Fig. 6(b), for example, we first calculated the $(5 \times 5) \mathrm{R}$ and $\mathrm{L}$ matrices of the complete configuration and then enforced the fact that the four conductors surrounding the central one are all kept on reference potential. The steps to be taken to enforce this common reference potential are the following. First, instead of working with the potentials as defined in (25), potential differences are introduced whereby the potential of one of the conductors surrounding the central one, say, conductor 5 (see numbering on the figure) is taken as the reference. If this potential is denoted by $V_{5}$, this implies that we introduce the differences $V_{i}-V_{5}, i=1,2,3,4$. At the same time, we have to enforce that the total current flowing back through 


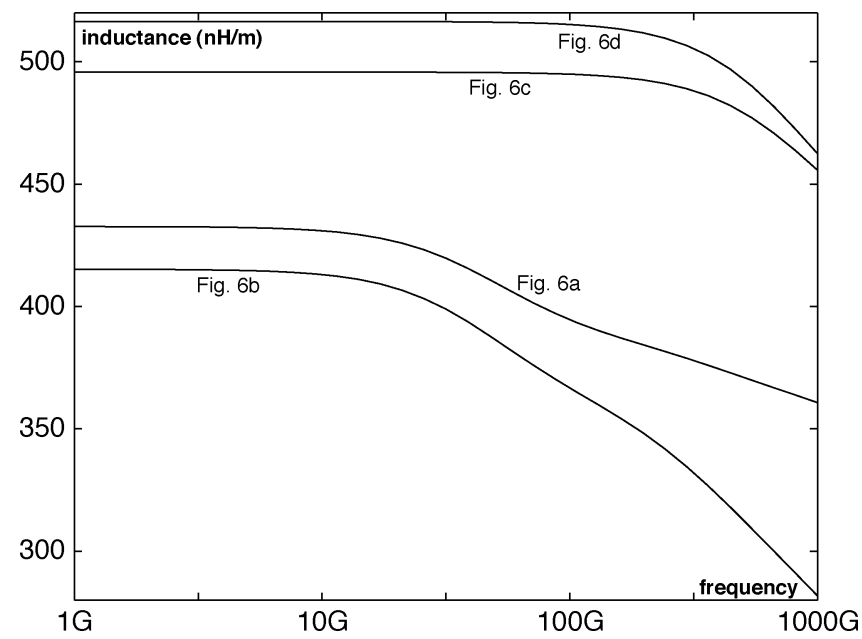

Fig. 8. Inductance PUL in nanohenry/meter as a function of frequency for the configurations of Fig. 6.

the reference conductor equals the sum of the currents flowing through the other ones, i.e., $\sum_{i=1}^{5} I_{i}=0$. As a consequence of this procedure, the original $5 \times 5 \mathrm{R}$ and $\mathrm{L}$ matrices are now reduced to $4 \times 4$ matrices, and this of course is also the case for the corresponding impedance matrix. The physical reason behind this procedure is that in the integral equation, the potentials are defined with respect to infinity, implying that current can flow back at infinity. In reality, only potential differences are meaningful. To complete the procedure, we still have to enforce the reference potential on conductors 2,3 , and 4 . The easiest way to do this is to invert the $4 \times 4$ impedance matrix $Z$ to obtain the currents as a function of the voltages. Now the voltages of conductors 2,3 , and 4 can easily be put to zero, and what remains is the voltage-current relation for the central conductor 1. This is the relationship displayed in Figs. 7 and 8. At this point, it is necessary to remark that to apply (23) and (24) to calculate the resistance and inductance data for the example of Fig. 1, the Green's function kernel $(1 / 2 \pi) \ln \left|\mathbf{r}-\mathbf{r}^{\prime}\right|$ is replaced by $(1 / 2 \pi) \ln \left|\mathbf{r}-\mathbf{r}^{\prime}\right|-(1 / 2 \pi) \ln \left|\mathbf{r}-\mathbf{r}^{\prime \prime}\right|$, where $\mathbf{r}^{\prime \prime}$ is the mirror image of $\mathbf{r}^{\prime}$ with respect to the PEC ground plane in Fig. 1. This automatically ensures that the presence of the PEC plane is taken into account and that the potentials are referenced to this ground plane.

Let us now return to Figs. 7 and 8. For the resistance results, we clearly observe the transition from the dc case to skin effect behavior. For the inductance results, we observe the decrease of the total inductance for increasing frequencies as the magnetic field is forced out of the conductors. However, even at the highest frequency of $1000 \mathrm{GHz}$, the skin depth is still $74.65 \mathrm{~nm}$ and the contribution from the so-called internal inductance is still important. This implies that the total inductance will further decrease when the frequency increases. The accuracy of the dc-resistance (which is the value obtained below $1 \mathrm{GHz}$ ) is better than $0.1 \%$ (case $1: 6.1728 \times 10^{5} \Omega / \mathrm{m}$, case 2: $6.1669 \times 10^{5} \Omega / \mathrm{m}$, case 3: $7.0416 \times 10^{5} \Omega / \mathrm{m}$, case 4: $\left.6.7901 \times 10^{5} \Omega / \mathrm{m}\right)$. To complete the data, Figs. 9 and 10 display all the elements of the original $5 \times 5 \mathrm{R}$ and $\mathrm{L}$ matrices for the configuration of Fig. 6(b), again as a function of frequency. We refer the reader to Fig. 6(b) for the numbering

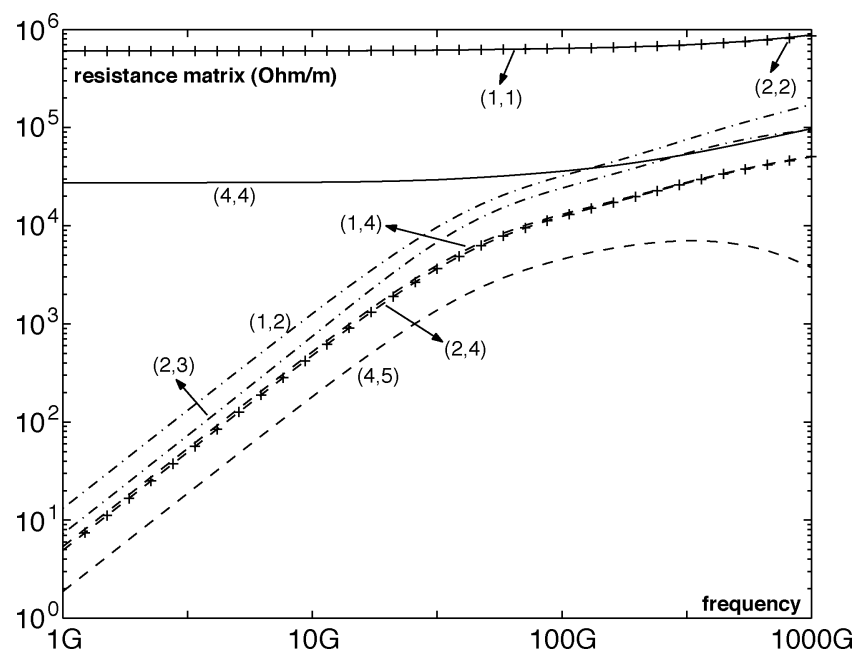

Fig. 9. Resistance matrix elements in $\Omega / \mathrm{m}$ as a function of frequency for the configuration of Fig. 6(b).

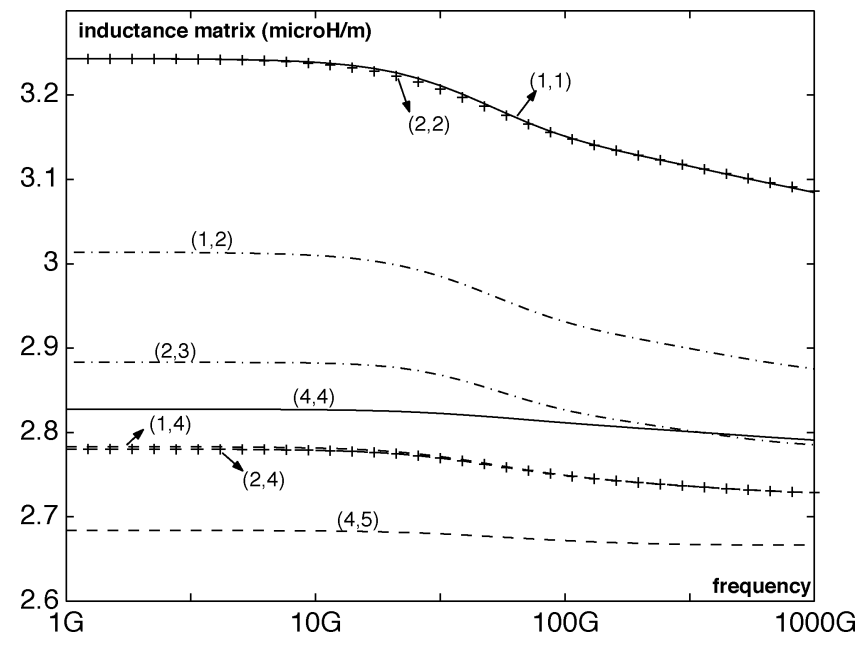

Fig. 10. Inductance matrix elements in microhenry/meter as a function of frequency for the configuration of Fig. 6(b).

of the conductors. Due to the symmetry of the configuration, only eight out of the 25 matrix elements are different (e.g. elements $(2,4),(4,2),(2,5),(5,2),(3,4),(4,3),(3,5)$, and $(5,3)$ are identical).

For a complete transmission line model, resistance and inductance data must be complemented with capacitance data. In the approximation used in this section, potentials are constant on each conductor. This implies that the relevant (frequency independent) capacitance matrices can be found by considering PEC conductors. The results given below are obtained using the approach described in [10]. As for the resistances and inductances, the capacitance matrices must be transformed into a single number, i.e., the capacitance PUL of the central signal line with respect to the surrounding "ground" conductors. The final capacitance values still depend on the dielectric properties of the medium in which the conductors are embedded. In ITRS and [9], several options are left open. The effective di-

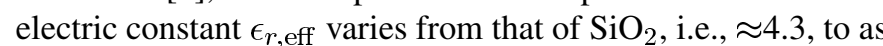
low as 2.9 for new low-k materials. As the background medium is modeled as a homogeneous dielectric, the capacitance will be 


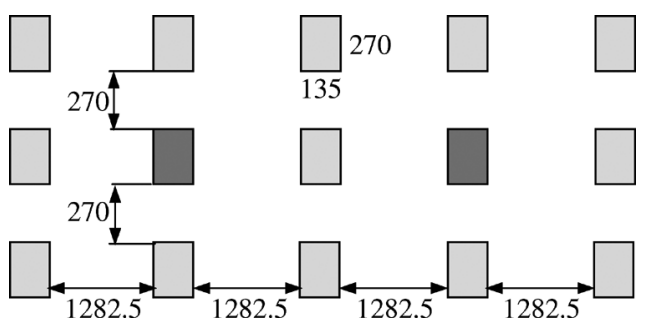

Fig. 11. Coupled signal line configuration based on Fig. 6(d).

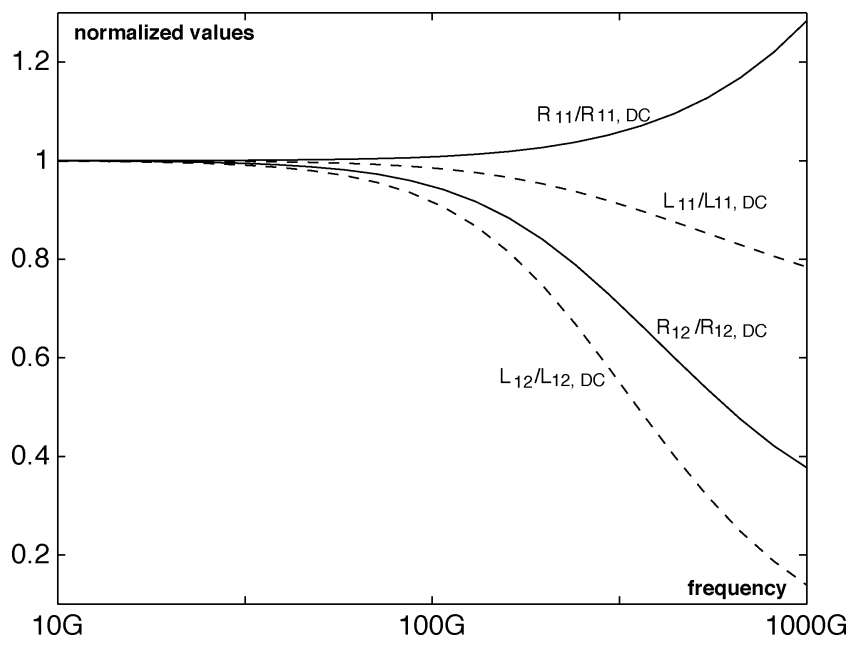

Fig. 12. Normalized resistance and inductance matrix elements as a function of frequency for the configuration of Fig. 11.

proportional to $\epsilon_{r, \text { eff }}$ and it suffices to give the capacitances for $\epsilon_{r, \text { eff }}=1$. For the configurations of Fig. 6(a)-(d), the following results were found: $C_{a}=38.35 \mathrm{pF} / \mathrm{m}, C_{b}=63.17 \mathrm{pF} / \mathrm{m}$, $C_{c}=34.79 \mathrm{pF} / \mathrm{m}$, and $C_{d}=38.20 \mathrm{pF} / \mathrm{m}$.

As a final example, consider the coupled signal line configuration of Fig. 11 based upon the configuration of Fig. 6(b). All conductors play the role of "ground" conductors except the two central ones. In this case, we obtain a $2 \times 2 \mathrm{R}$ and $\mathrm{L}$ matrix. These matrices are symmetrical $\left(R_{12}=R_{21}, L_{12}=L_{21}\right)$ and due to the symmetry of the geometry, the diagonal elements are also identical $\left(R_{11}=R_{22}, L_{11}=L_{22}\right)$. Fig. 12 shows the variation of the normalized elements of these matrices as a function of frequency, i.e., $R_{11} / R_{11, \mathrm{DC}}, R_{12} / R_{12 \text {,DC }}, L_{11} / L_{11, \mathrm{DC}}$ and $L_{12} / L_{12, \mathrm{DC}}$. We have chosen to display the normalized values in order to obtain a clear graphical representation. The dc values are: $R_{11, \mathrm{DC}}=6.5000 \times 10^{5} \Omega / \mathrm{m}, R_{12, \mathrm{DC}}=4.6427 \times$ $10^{4} \Omega / \mathrm{m}, L_{11, \mathrm{DC}}=599.0 \mathrm{nH} / \mathrm{m}$, and $L_{12, \mathrm{DC}}=-89.61 \mathrm{nH} / \mathrm{m}$. The normalized values differ less than $0.1 \%$ from unity below $10 \mathrm{GHz}$. One can readily derive that $R_{11, \mathrm{DC}}=(14 / 13) R$ and $R_{12, \mathrm{DC}}=(1 / 13) R$, with $R$ the dc resistance PUL of one of the conductors, i.e., $R=\rho_{\text {eff }} /(w t)$ with $w=135 \mathrm{~nm}, t=270 \mathrm{~nm}$, and $\rho_{\text {eff }}=2.2 \mu \mathrm{m} \cdot \Omega \cdot \mathrm{cm}$. Further remark that $L_{12, \mathrm{DC}}$ is negative, but this is physically acceptable.

\section{CONCLUSION}

In this paper, it was shown that a recently introduced surface admittance concept to model the current crowding and the skin effect for rectangular conductors in the 2-D TM case is ideally suited to predict the behavior of future on-chip signal line configurations as proposed by the ITRS. Details were given on the relevant electric field integral equation and on the way to obtain the correct resistance and inductance data. Furthermore, starting from the 2-D model, a 1-D local admittance model with coupled currents at the top and bottom of a general 3-D conductor was derived. It was shown, both theoretically and through a numerical example, that this new admittance model performs better over the whole frequency range compared to an often used local impedance formula separately applied to the top and bottom currents. Future research aims at extending the proposed concepts to three-dimensional configurations.

\section{REFERENCES}

[1] T. K. Sarkar and A. R. Djordjević, "Wideband electromagnetic analysis of finite conductivity cylinders," Progr. Electromagn. Res., vol. 16, pp. 153-173, 1997.

[2] G. Antonini, A. Orlandi, and C. R. Paul, "Internal impedance of conductors of rectangular cross section," IEEE Trans. Microwave Theory Tech., vol. 47, no. 7, pp. 979-985, Jul. 1999.

[3] K. M. Coperich, A. Ruehli, and A. Cangellaris, "Enhanced skin effect for partial-element equivalent-circuit (PEEC) models," IEEE Trans. Microwave Theory Tech., vol. 48, no. 9, pp. 1435-1442, Sep. 2000.

[4] D. De Zutter and L. Knockaert, "Skin effect modeling based on a differential surface admittance operator," IEEE Trans. Microwave Theory Tech., vol. 53, no. 8, pp. 1199-1206, Aug. 2005.

[5] R. Horton, B. Easter, and A. Gopinath, "Variation of microstrip losses with thickness of strip," Electron. Lett., vol. 7, no. 17, pp. 490-491, Aug. 1971.

[6] C. L. Holloway, Edge and surface effects on conductor loss associated with planar circuits MIMICAD Tech. Rep. 12, Apr. 1992.

[7] E. Tuncer, B.-T. Lee, M. S. Islam, and D. P. Neikirk, "Quasistatic conductor loss calculation in transmission lines using a new conformal mapping technique," IEEE Trans. Microwave Theory Tech., vol. 42, no. 9, pp. 1807-1815, Sep. 1994.

[8] F. Olyslager and D. De Zutter, "Skin effect," in Encyclopedia of RF and Microwave Engineering, K. Chang, Ed. New York: Wiley, 2005, vol. 5, pp. 4669-4675.

[9] IST, Compact modelling of on-chip passive structures at high frequencies IST-2001-34058 project, 2004.

[10] F. Olyslager, N. Faché, and D. De Zutter, "New fast and accurate line parameter calculation of general multiconductor transmission lines in multilayered media," IEEE Trans. Microwave Theory Tech., vol. 39, no. 6, pp. 901-909, Jun. 1991.

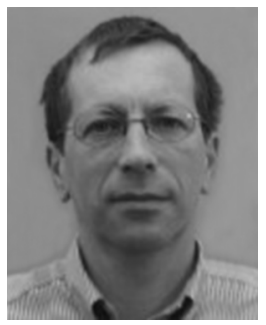

Daniël De Zutter (M'92-SM'96-F'01) was born in 1953. He received the M.Sc. degree in electrical engineering and the Ph.D. degree from Ghent University, Ghent, Belgium, in 1976 and 1981, respectively. In 1984, he completed a thesis leading to a degree equivalent to the French Aggrégation or the German Habilitation.

From 1984 to 1996, he was with the National Fund for Scientific Research of Belgium. He is now a full Professor of electromagnetics, Ghent University. Most of his earlier scientific work dealt with the electrodynamics of moving media. His research now focuses on all aspects of circuit and electromagnetic modelling of high-speed and high-frequency interconnections and packaging, electromagnetic compatibility, and numerical solutions of Maxwell's equations. As author or coauthor he has contributed to more than 140 international journal papers and 150 papers in conference proceedings.

Prof. De Zutter (with F. Olyslager and K. Blomme) received the 1995 IEEE Microwave Prize Award from the IEEE Microwave Theory and Techniques Society. In 1999 he received the Transactions Prize Paper Award from the IEEE EMC Society. At present, he is an Associate Editor for the IEEE TRANSACTIONS ON MICROWAVE THEORY AND TECHNIQUES. 


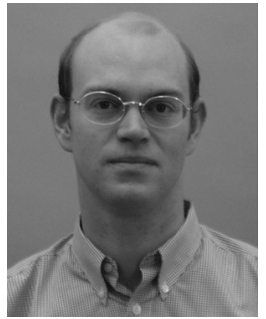

Hendrik Rogier (M'00-SM'06) was born in 1971. $\mathrm{He}$ received the electrical engineering and Ph.D. degrees from Ghent University, Ghent, Belgium, in 1994 and 1999, respectively.

He is currently a Postdoctoral Research Fellow with FWO-V and a part-time Professor with the Department of Information Technology, Ghent University. From October 2003 to April 2004, he was a Visiting Scientist with the Mobile Communications Group, Vienna University of Technology. $\mathrm{He}$ has authored or coauthored about 30 papers in international journals and about 45 contributions in conference proceedings. His current research interests are the analysis of electromagnetic waveguides, electromagnetic simulation techniques applied to electromagnetic compatibility and signal integrity problems, as well as to indoor propagation and antenna design, and smart antenna systems for wireless networks.

Dr. Rogier received the URSI Young Scientist Award at the 2001 URSI Symposium on Electromagnetic Theory and at the 2002 URSI General Assembly.

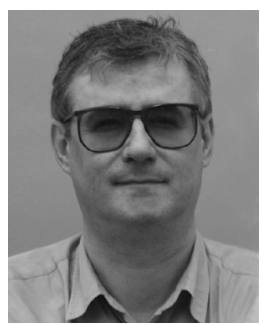

Luc Knockaert (M'81-SM'00) received the M.Sc. degree in physical engineering, the M.Sc. degree in telecommunications engineering, and the Ph.D. degree in electrical engineering from Ghent University, Ghent, Belgium, in 1974, 1977, and 1987, respectively.

From 1979 to 1984 and from 1988 to 1995 , he worked on north-south cooperation and development projects at the Universities of Congo and Burundi. $\mathrm{He}$ is presently a Professor at the Department of Information Technology, Ghent University, and Senior Researcher with IMEC. His current interests are the application of linear algebra and adaptive methods in signal estimation and model order reduction in computational electromagnetics. As author or coauthor he has contributed to about 100 international journal and conference publications.

Dr. Knockaert is a member of MAA and SIAM.

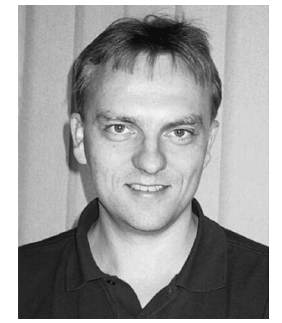

Jeannick Sercu received the degree in electrical engineering and the Ph.D. degree from Ghent University, Ghent, Belgium, in 1990 and 1994, respectively.

From October 1994 to September 1996, he was a Postdoctoral Fellow at the Department of Information Technology, University of Ghent. His research dealt with full-wave electromagnetic simulation of planar structures in multilayered media. His work was sponsored by the National Fund for Scientific Research in Belgium and the Flemish Institute for the Scientific and Technological Research in the Industry. In October 1996, he joined the EEsof EDA division, Agilent Technologies (formerly Hewlett Packard), as a Software Design Research and Development Engineer, working for the electromagnetic (EM) project team. He made contributions within the domain of EM and circuit simulation and worked on momentum microwave and RF electromagnetic engines, physical spice modeling, nonlinear modeling, and physical layout design and modeling. $\mathrm{He}$ is the author or coauthor of more than 30 technical papers in international journals and in proceedings of international conferences. In February 2001, he obtained a Technical Lead position as Research and Development Expert within the EEsof EDA division, Agilent Technologies, focusing on physical simulation and verification. He has received patents. 